\title{
Correction to: A Prediction of Heart Disease Using Machine Learning Algorithms
}

Mohd Faisal Ansari, Bhavya Alankar, and Harleen Kaur

\section{Correction to:}

Chapter "A Prediction of Heart Disease Using Machine

Learning Algorithms" in: J. I.-Z. Chen et al. (Eds.):

Image Processing and Capsule Networks, AISC 1200,

https://doi.org/10.1007/978-3-030-51859-2_45

The original version of the book was inadvertently published with an incorrect author name in chapter 45 . The chapter and book have now been updated with the changes. 